\title{
Clinical Evaluation of the Gen-Probe Amplified Mycobacterium Tuberculosis Direct Test for Rapid Diagnosis of Tuberculosis Lymphadenitis
}

\author{
A. Kerleguer, ${ }^{1}$ M. Fabre, ${ }^{2}$ J. J. Bernatas, ${ }^{3}$ P. Gerome, ${ }^{4}$ E. Nicand, ${ }^{1}$ V. Herve ${ }^{2}$ \\ and J. L. Koeck ${ }^{1 *}$ \\ Laboratoire de Biologie Clinique, HIA Val-de-Grāce, Paris, ${ }^{1}$ Laboratoire de Biologie Clinique, HIA Percy, Clamart, ${ }^{2}$ \\ Laboratoire de Biologie Clinique, HIA Desgenettes, Lyon, ${ }^{4}$ France, and Centre Paul Faure, Djibouti, \\ Republic of Djibouti ${ }^{3}$
}

Received 17 October 2003/Returned for modification 17 November 2003/Accepted 20 July 2004

\begin{abstract}
This prospective study evaluated the performance of the Amplified Mycobacterium Tuberculosis Direct Test (MTD) for the diagnosis of lymph node tuberculosis in Djibouti, Republic of Djibouti. Of 197 specimens sampled from 153 patients, 123 were from 95 tuberculous patients. The sensitivity and specificity of MTD were 93 and $100 \%$, respectively. The sensitivity of culture was $89 \%$.
\end{abstract}

Because of its high incidence, tuberculosis is probably the major cause of death in the Republic of Djibouti, a small country located in the Horn of Africa. Moreover, lymph node (LN) tuberculosis represents about $25 \%$ of all the clinical forms of tuberculosis. LN specimens, like other extrapulmonary specimens, contain few tubercle bacilli, leading to a low sensitivity of smear microscopy for detection of acid-fast bacilli; paucibacillary tuberculosis requires a much longer incubation time for positive growth (9). With the development of novel techniques in molecular biology, these delays might be shortened. The Amplified Mycobacterium Tuberculosis Direct Test (MTD) detects specific Mycobacterium tuberculosis RNA by using an isothermal transcription-mediated amplification method. MTD has been shown to be a sensitive, specific, and rapid method for detecting $M$. tuberculosis in clinical samples (6). We evaluated the clinical use and performance of this test for the rapid diagnosis of LN tuberculosis.

From January to April 1999, patients attending the Paul Faure clinic (a center specializing in tuberculosis diagnosis and treatment) in Djibouti, Republic of Djibouti, and presenting an $\mathrm{LN}$ with a diameter of $>10 \mathrm{~mm}$ were included in the study. A standardized questionnaire was filled out for each patient. Specimens were collected by fine-needle (18-gauge) aspiration of the LN and then collected in a sterile tube. If the volume of the specimen was $<0.1 \mathrm{ml}$, the needle and syringe were rinsed with $1 \mathrm{ml}$ of sterile water. All specimens were shipped at room temperature (using air carrier) to Paris, France, and generally arrived within $48 \mathrm{~h}$ of collection. All analyses of specimens were performed in Paris, France. After decontamination using the $N$-acetyl-L-cysteine-sodium hydroxide procedure, specimens were separated into two equal parts. The first part was used for microscopy and culture (solid media, LowensteinJensen and Coletsos; liquid medium, Mycobacterial Growth

\footnotetext{
* Corresponding author. Present address: Laboratoire de Biologie Clinique, HIA Robert Picque, 351 route de Toulouse, Bordeaux 33140, France. Phone: 335568 47098. Fax: 335568 47495. E-mail: labos.hiarp@wanadoo.fr.
}

Indicator Tube [MGIT]). The second part was used to perform MTD. The remainder of the specimen was stored.

Isolates of $M$. tuberculosis complex strains were identified by routine biochemical methods and DNA probes (Accuprobe; Gen-Probe, Inc., San Diego, Calif.). The cutoff value for a positive MTD result was set at 1,000,000 relative light units (RLU), and the equivocal zone was defined as values between 30,000 and 1,000,000 RLU (1, 5). Each run included positive and negative amplification controls. The positive amplification control was prepared from a $10^{-4}$ to $10^{-5}$ dilution of a suspension of $M$. tuberculosis with a McFarland standard of 1 . Sterile water was used as the negative amplification control. The run was validated when the negative-control value was $<20,000 \mathrm{RLU}$ and the positive-control value was $>500,000$ RLU.

All specimens were analyzed for the presence of amplification inhibitors by adding an aliquot of the positive control to the sample. A $1 / 5$ dilution in sterile water was used to remove inhibitors. If there was a discrepancy between culture and MTD results, the final diagnosis was made by considering the patient's clinical, biological, and radiological findings (8); the factors taken into account were a positive culture for another sample from the same patient, clinical signs with highly suggestive lesions on chest X-ray, a known history of tuberculosis, or a favorable outcome with antituberculosis treatment. Assay performance was evaluated by comparing the assay results to the final diagnosis.

From January 1999 to April 1999, 197 LN aspiration specimens from various sites (cervical, 98; submaxillar, 42; axillar, 21; supraclavicular, 19; inguinal, 10; others, 7) were obtained from 153 patients. Of the 197 specimens, 30 were smear positive and culture positive, 89 were smear negative and culture negative, and 78 were smear negative and culture positive. A total of 108 strains belonging to the $M$. tuberculosis complex were isolated (M. tuberculosis [89.9\%], Mycobacterium africanum [4.6\%], Mycobacterium canetti [4.6\%], and Mycobacterium bovis [0.9\%]). No atypical Mycobacterium species was isolated.

Of the 115 samples testing positive with MTD, 30 were smear and culture positive, 70 were smear negative and culture 
positive, and 15 were smear and culture negative. Of the 82 MTD-negative samples, none were smear and culture positive, 8 were smear negative and culture positive, and 74 were smear and culture negative. Four samples $(2 \%)$ exhibited amplification inhibition (two culture-negative samples and two culturepositive samples; for the latter, inhibitors were successfully removed after sample dilution).

The eight false-negative specimens (MTD negative and culture positive) represent $7.4 \%$ (8 of 108) of the culture-positive samples; they did not contain amplification inhibitors. Some researchers have noted that, in contrast to pulmonary specimens, the lack of sensitivity of molecular diagnosis performed on extrapulmonary samples might result from the use of very small sample volumes and an irregular dispersion of the aggregated bacteria in paucibacillary specimens $(4,8)$. Our data are in accord with this hypothesis: seven of the eight discrepant samples had a very low volume $(<0.1 \mathrm{ml})$, four samples had discrepancies between the culture medium used (three samples gave a negative result in MGIT and a positive result on solid medium, and one sample gave a positive result in MGIT and a negative result on solid medium), the time to culture positivity was high, and the number of colonies on slants was low (between one and three colonies per two tubes for seven samples). The 15 apparent false-positive specimens with MTD (MTD positive, culture negative) came from patients with confirmed tuberculosis, according to clinical, historical, and other biological findings (respiratory tuberculosis, 2 patients; positive response to treatment, 3 patients; history of tuberculosis, 3 patients; other $\mathrm{LN}$ with positive culture, 4 patients).

After the discrepant results were resolved, of the 153 total patients assessed, $95(62 \%)$ had a conclusive diagnosis of tuberculosis. The sensitivity, specificity, positive predictive value, and negative predictive value for the 197 specimens tested were $87,100,100$, and $83 \%$ for culture, respectively, and 93, 100,100 , and $90 \%$ for MTD, respectively (Table 1 ).

Other researchers have evaluated the performance of MTD for diagnosis of LN tuberculosis on a limited number of samples $(<40)$. The specificity of MTD approached $100 \%(2,3)$, but its sensitivity was more variable, ranging from $77.8 \%$ (3) to $92.8 \%$ (2). For extrapulmonary specimens, the overall sensitivity of MTD ranged from 74.3 to $100 \%$ (7). In another study, we reported similar results for pulmonary samples (5). The results presented here suggest that irregular dispersion of tubercle bacilli in a specimen could diminish the sensitivity of both MTD and culture. However, the sensitivity of MTD was superior to that of culture. Therefore, culture cannot be considered the yardstick for the measurement of molecular biology test sensitivity (7) and should be evaluated versus a "gold standard" built with clinical, radiological, and biological findings.

Given the high performance of MTD with fine-needle LN
TABLE 1. Performance of culture and MTD on LN specimens after discrepant results were resolved

\begin{tabular}{|c|c|c|c|}
\hline \multirow{2}{*}{$\begin{array}{l}\text { Method and } \\
\quad \text { result }^{a}\end{array}$} & \multicolumn{3}{|c|}{$\begin{array}{l}\text { No. of LN specimens with a conclusive diagnosis } \\
\text { of tuberculosis }\end{array}$} \\
\hline & $\begin{array}{l}\text { Positive } \\
(n=123)\end{array}$ & $\begin{array}{l}\text { Negative } \\
(n=74)\end{array}$ & $\begin{array}{c}\text { Total } \\
(n=197)\end{array}$ \\
\hline \multicolumn{4}{|l|}{ Culture } \\
\hline Positive & 108 & 0 & 108 \\
\hline Negative & 15 & 74 & 89 \\
\hline \multicolumn{4}{|l|}{ MTD } \\
\hline Positive & 115 & 0 & 115 \\
\hline Negative & 8 & 74 & 82 \\
\hline
\end{tabular}

${ }^{a}$ For culture, the sensitivity was $87 \%$, the specificity was $100 \%$, the positive predictive value was $100 \%$, and the negative predictive value was $83 \%$. For MTD, the sensitivity was $93 \%$, the specificity was $100 \%$, the positive predictive value was $100 \%$, and the negative predictive value was $90 \%$.

aspiration specimens, we recommend this technique for a rapid diagnosis of LN tuberculosis. The use of MTD and fine-needle $\mathrm{LN}$ aspiration could reduce the necessity for performing open LN biopsy when testing for LN tuberculosis; it could also be used in developing countries to perform epidemiological surveys.

\section{REFERENCES}

1. Alcala, L., M. J. Ruiz-Serrano, S. Hernangomez, M. Marin, D. G. de Viedma, R. San Juan, and E. Bouza. 2001. Evaluation of the upgraded Amplified Mycobacterium Tuberculosis Direct Test (Gen-Probe) for the direct detection of Mycobacterium tuberculosis in respiratory and non-respiratory specimens. Diagn. Microbiol. Infect. Dis. 41:51-56.

2. Ehlers, S., R. Ignatius, T. Regnath, and H. Hahn. 1996. Diagnosis of extrapulmonary tuberculosis by Gen-Probe Amplified Mycobacterium tuberculosis Direct Test. J. Clin. Microbiol. 34:2275-2279.

3. Gamboa, F., G. Fernandez, E. Padilla, J. M. Manterola, J. Lonca, P. J. Cardona, L. Matas, and V. Ausina. 1998. Comparative evaluation of initial and new versions of the Gen-Probe Amplified Mycobacterium Tuberculosis Direct Test for direct detection of Mycobacterium tuberculosis in respiratory and nonrespiratory specimens. J. Clin. Microbiol. 36:684-689.

4. Ieven, M., and H. Goossens. 1997. Relevance of nucleic acid amplification techniques for diagnosis of respiratory tract infections in the clinical laboratory. Clin. Microbiol. Rev. 10:242-256.

5. Kerleguer, A., J. L. Koeck, M. Fabre, P. Gérôme, R. Teyssou, and V. Hervé. 2003. Use of equivocal zone in interpretation of results of the Amplified Mycobacterium Tuberculosis Direct Test for diagnosis of tuberculosis. J. Clin. Microbiol. 41:1783-1784

6. Piersimoni, C., C. Scarparo, P. Piccoli, A. Rigon, G. Ruggerio, D. Nista, and S. Bornigia. 2002. Performance assessment of two commercial amplification assays for direct detection of Mycobacterium tuberculosis complex from respiratory and extrapulmonary specimens. J. Clin. Microbiol. 40:4138-4142.

7. Piersimoni, C., and C. Scarparo. 2002. Relevance of commercial amplification methods for direct detection of Mycobacterium tuberculosis complex in clinical samples. J. Clin. Microbiol. 41:5355-5365.

8. Scarparo, C., P. Piccoli, A. Rigon, G. Ruggiero, M. Scagnelli, and C. Piersimoni. 2000. Comparison of enhanced Mycobacterium tuberculosis Amplified Direct Test with COBAS AMPLICOR Mycobacterium tuberculosis Assay for direct detection of Mycobacterium tuberculosis complex in respiratory and extrapulmonary specimens. J. Clin. Microbiol. 38:1559-1562.

9. Sepkowitz, K. A., J. Raffalli, L. Riley, T. E. Kiehn, and D. Armstrong. 1995 Tuberculosis in the AIDS era. Clin. Microbiol. Rev. 8:180-199. 